\title{
Stability of liquid bridges between an elliptical and a circular supporting disk
}

\author{
A. Laverón-Simavilla, J. Meseguer, and J. L. Espino \\ IDR/UPM, E.T.S.I. Aeronáuticos, Universidad Politécnica de Madrid, E-28040 Madrid, Spain
}

(Received 27 February 2003; accepted 24 June 2003; published 15 August 2003)

\begin{abstract}
A numerical method has been developed to determine the stability limits for liquid bridges held between noncircular supporting disks, and the application to a configuration with a circular and an elliptical disk subjected to axial acceleration has been made. The numerical method led to results very different from the available analytical solution, which has been revisited and a better approximation has been obtained. It has been found that just retaining one more term in the asymptotic analysis the solution reproduces the real behavior of the configuration and the numerical results. () 2003 American Institute of Physics. [DOI: 10.1063/1.1601615]
\end{abstract}

\section{INTRODUCTION}

The study of liquid bridges behavior started more than a century ago. However, interest in their response has been continuously growing during the last decades due to different applications as the floating-zone technique for crystal growth, the measurement of the surface tension, shear viscosity and extensional viscosity of molten Newtonian and non-Newtonian liquids, and the agglomeration of particles, among others. Concerning the floating-zone technique, liquid bridges offer the simplest, ${ }^{1,2}$ while still representative, model of the mechanical behavior of this crystal growing technique. In this technique, a liquid zone is established by melting an initially solid rod where the molten region is held between two still solid parts of the rod. One part plays the role of a charge or feed material (typically polycrystalline) and on the other solid part a single crystal is grown by translating the rod through a temperature gradient so as to melt the feed rod and solidifying a crystal from the molten zone.

The nominal configuration considered in the literature is a cylindrical shape held between equal circular disks. The equilibrium shapes and stability limits of the mentioned configuration subjected to various disturbances, that can act either accidentally or intentionally, is a matter of great concern. Most of these studies deal with the stability of liquid bridges held between circular disks and subjected to an acceleration field. ${ }^{3,4}$ Some effort has also been devoted to analyze the influence of other perturbations that can be used to modify the stability of the liquid bridge configuration (for example, by using flow stabilization, ${ }^{5}$ acoustic stabilization, ${ }^{6}$ electric field stabilization, ${ }^{7}$ magnetic field stabilization, ${ }^{8}$ or thermal convection ${ }^{9,10}$ ).

The influence of different perturbations on the stability of liquid bridges have been extensively analyzed from both the theoretical and the experimental point of view. However, leaving apart nonmechanical disturbances, except in a few papers very recently published (two of them dealing with the equilibrium interface shapes of liquid bridges between noncircular supports, ${ }^{11,12}$ and a third dealing with the asymptotic stability of long liquid bridges between noncircular disks ${ }^{13}$ ), to our knowledge all published works to date deal with liquid bridge configurations (either axisymmetric or nonaxisymmetric) that are held between circular supports. Therefore, except for the above quoted papers, the effects of noncircular supports have never been considered, and no efforts have been devoted to the study of the behavior of liquid bridges held between noncircular supports (although some early work concerning capillary jets emerging from noncircular nozzles has been published ${ }^{14}$ ).

In the above quoted asymptotic stability analysis, ${ }^{13}$ it is demonstrated that the influence on the stability limit of perturbations like an axial gravity and the existence of noncircular supporting disks can somehow interact in the way of balancing each other. The destabilizing effect of an axial gravity can be counteracted by placing the liquid column between noncircular disks, and this compensation will take place irrespective of the values of the parameters involved, provided the resulting configuration is stable. Obviously this behavior is derived from an asymptotic study, so that one cannot expect that in a real situation the values of both gravity and the parameter measuring the eccentricity can be increased without any further limitation and the results will still be valid.

A numerical method capable of solving the problem without any asymptotic approximation is implemented in Sec. III. This method was already used for analyzing stability problems of nonaxisymmetric liquid bridges held between circular disks, ${ }^{15,16}$ but it is here adapted to the particularities imposed by the nonaxisymmetric boundary conditions.

In Sec. IV the asymptotic stability approximation published by Meseguer et al. ${ }^{13}$ is revisited, and higher-order terms dealing with the combined effect of both axial acceleration and noncircular disks are added to the bifurcation equation. The bifurcation equation already known ${ }^{13}$ is therefore substituted by a more accurate expression, the effect being a significant reduction of the stable region, in great accordance with the numerical results.

Due to the infinite possible disk shapes that can be considered, and to keep the effort in the analysis within reasonable limits, still obtaining conclusions, it is assumed that one 
of the disks is circular and the other disk is elliptical, its mean radius being equal to the radius of the circular disk. If any other mean radius were used, the effect of the difference with the other disk's mean radius would be much larger than the effect of having a noncircular shape and would mask the aspect analyzed.

\section{FORMULATION}

The liquid bridge model considered here consists of an isothermal mass of liquid of constant properties (the difference in density between the liquid bridge and the surrounding fluid, $\Delta \rho$, and surface tension, $\sigma$ ), held by surface tension forces between two parallel, one circular and the other elliptical, solid disks and subjected to an axial acceleration, $\mathbf{g}$ $=-g \mathbf{k}$. It is therefore assumed that $g$ is positive when the axial acceleration is directed from the upper to the lower disk, and $g$ is negative if the acceleration is directed from the lower to the upper disk. The lower disk is circular with radius $R_{0}^{-}$and the upper disk is elliptical with semiaxes equal to $R_{0}^{+}(1+a)$ and $R_{0}^{+}(1-a)$, respectively. Both disks are coaxial and they are separated by a distance $L$ (see Fig. 1 ), and $\tilde{V}$ is the volume of the liquid drop spanning between both disks.

Equilibrium shapes of liquid bridges are described by the Young-Laplace equation, which in dimensional variables takes the form

$$
\sigma \tilde{M}(\tilde{F})+\widetilde{P}-\Delta \rho g \tilde{z}=0,
$$

where $\tilde{M}(\tilde{F})$ is twice the mean curvature of the interface, $\widetilde{F}=\widetilde{F}(\widetilde{z}, \theta)$ is the equation for the liquid bridge interface, and $\widetilde{P}$ is a constant related to the origin of pressure. The boundary conditions and constraints express the azimuthal periodicity of the interface shape, $\widetilde{F}(\widetilde{z}, \theta)=\widetilde{F}(\widetilde{z}, \theta+2 \pi)$, the constant value of the liquid bridge volume, and that the bridge surface is anchored or pinned to the edges of the supporting disks.

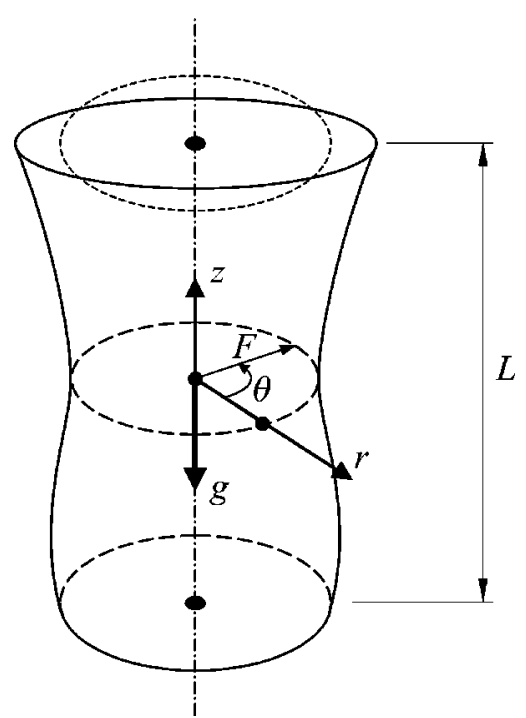

FIG. 1. Sketch of the considered configuration. The lower disk is a circle while the upper disk is bounded by an ellipse.

Let, in a general case, $\widetilde{F}(L / 2, \theta)$ and $\tilde{F}(-L / 2, \theta)$ be the shape of the supporting disks. If the following characteristic lengths, associated to the respective disk shape, are introduced:

$$
\begin{aligned}
& R^{+}=\frac{1}{2 \pi} \int_{0}^{2 \pi} \tilde{F}(L / 2, \theta) \mathrm{d} \theta, \\
& R^{-}=\frac{1}{2 \pi} \int_{0}^{2 \pi} \tilde{F}(-L / 2, \theta) \mathrm{d} \theta,
\end{aligned}
$$

then, taking $R=\left(R^{+}+R^{-}\right) / 2$ as the characteristic length of the problem, and defining $F=\tilde{F} / R, z=\tilde{z} / R$, Eq. (1) can be written as

$$
M(F)+P-B z=0,
$$

where $\widetilde{P}$ has been made dimensionless using $\sigma / R, B$ is the axial Bond number, $B=\Delta \rho g R^{2} / \sigma$, and

$$
M(F)=\frac{F\left[1+\left(F_{z}\right)^{2}\right]\left(F_{\theta \theta}-F\right)+F F_{z z}\left[F^{2}+\left(F_{\theta}\right)^{2}\right]-2 F_{\theta}\left(F_{\theta}+F F_{z} F_{z \theta}\right)}{\left\{F^{2}\left[1+\left(F_{z}\right)^{2}\right]+\left(F_{\theta}\right)^{2}\right\}^{3 / 2}}
$$

is twice the dimensionless mean curvature. The subscripts $z$ and $\theta$ indicate derivatives with respect to these variables.

For the disk configuration considered in this paper [a circular disk of radius $R_{0}^{-}$and an elliptical disk with semiaxes equal to $R_{0}^{+}(1+a)$ and $R_{0}^{+}(1-a)$ ], the boundary conditions expressing that the bridge surface is anchored or pinned to the edges of the supporting disks are

$$
\begin{aligned}
& \tilde{F}(L / 2, \theta)=\frac{R_{0}^{+}\left(1-a^{2}\right)}{\left(1+a^{2}-2 a \cos 2 \theta\right)^{1 / 2}}, \\
& \tilde{F}(-L / 2, \theta)=R_{0}^{-} .
\end{aligned}
$$

To decouple the effect of noncircularity from the effect of mean radii difference, the following characteristic parameter associated to the upper disk shape is introduced:

$$
\tau_{R}=\frac{1}{2 \pi} \int_{0}^{2 \pi} \frac{1-a^{2}}{\left(1+a^{2}-2 a \cos 2 \theta\right)^{1 / 2}} \mathrm{~d} \theta
$$

and assuming that the upper disk mean radius is $1 / \tau_{R}$ times the radius of the lower disk, $R_{0}^{+}=R_{0}^{-} / \tau_{R}$, both disks will have the same characteristic radius: $R=R^{-}=R^{+}$. Otherwise, as already stated, the effect of the noncircularity of the disks would be masked by the effect of the different mean sizes of the disks. 
The boundary conditions at the disks can be expressed in dimensionless form as

$$
\begin{aligned}
& F(\Lambda, \theta)=\frac{\left(1-a^{2}\right)}{\tau_{R}\left(1+a^{2}-2 a \cos 2 \theta\right)^{1 / 2}}, \\
& F(-\Lambda, \theta)=1,
\end{aligned}
$$

whereas the azimuthal periodicity condition and the volume preservation condition are

$$
\begin{aligned}
& F(z, \theta+2 \pi)=F(z, \theta), \\
& \frac{1}{2} \int_{-\Lambda}^{\Lambda} \mathrm{d} z \int_{0}^{2 \pi} F^{2} \mathrm{~d} \theta=2 \pi \Lambda V .
\end{aligned}
$$

In the above expressions $V$ is the dimensionless volume, $V=\tilde{V} /\left(\pi R^{2} L\right)$, and $\Lambda=L /(2 R)$ is the slenderness. Although both the numerical and the asymptotical analysis allow us to consider different volumes, in the subsequent sections the volume of the liquid bridge will be fixed to that of the right cylinder of the same slenderness and radii equal to that of the circular supporting disk, which means $V=1$.

\section{NUMERICAL ANALYSIS}

In previous papers ${ }^{15,16}$ an algorithm, based on a continuation method ${ }^{17}$ capable of overpassing bifurcation points and turning points, was developed using a finite-difference method, and was used to obtain the bifurcation diagrams and equilibrium shapes of nonaxisymmetric liquid bridges subject to a lateral gravitational force, and to combined lateral and axial gravitational forces. In this section the algorithm is extended to liquid bridges held between noncircular disks in the presence of an axial gravitational field.

The method is based on linearizing the formulation [Eqs. (3), (6)-(8)] around a known solution $\left[F_{0}(z, \theta), P_{0}\right]$, by seeking solutions of the form

$$
\begin{aligned}
& F(z, \theta)=F_{0}(z, \theta)+f(z, \theta)+o\left(\left|\frac{f}{F_{0}}\right|\right), \\
& P=P_{0}+p+o\left(\left|\frac{p}{P_{0}}\right|\right),
\end{aligned}
$$

where $\left|f / F_{0}\right| \ll 1$ and $\left|p / P_{0}\right| \ll 1$. The leading terms obtained for Eq. (3) now give an equation for $f(z, \theta)$,

$$
\begin{aligned}
\widetilde{O}^{-3 / 2}\left[\tilde{A}+\left(\widetilde{B}-\frac{3 \tilde{A} \widetilde{Q}}{2 \widetilde{O}}\right) f+\left(\widetilde{C}-\frac{3 \widetilde{A} \widetilde{S}}{2 \widetilde{O}}\right) f_{z}+\left(\tilde{D}-\frac{3 \tilde{A} \widetilde{T}}{2 \widetilde{O}}\right) f_{\theta}\right] \\
+\widetilde{E} f_{z z}+\widetilde{G} f_{\theta \theta}+\widetilde{H} f_{z \theta}+P_{0}+p-B z=0,
\end{aligned}
$$

where $\widetilde{A}, \widetilde{B}, \widetilde{C}, \widetilde{D}, \widetilde{E}, \widetilde{G}, \widetilde{H}, \widetilde{O}, \widetilde{Q}, \widetilde{S}$, and $\widetilde{T}$ are known functions of $F_{0}(z, \theta)$ and, consequently, of the point considered on the interface.

The leading terms obtained for the boundary conditions and the constraint that determines the reference pressure are

$$
\begin{aligned}
& \int_{-\Lambda}^{\Lambda} \mathrm{d} z \int_{0}^{2 \pi} F_{0}(z, \theta)^{2} \mathrm{~d} \theta+2 \int_{-\Lambda}^{\Lambda} \mathrm{d} z \int_{0}^{2 \pi}\left[F_{0}(z, \theta) f(z, \theta)\right] \mathrm{d} \theta \\
& \quad=4 \pi \Lambda
\end{aligned}
$$

$F_{0}(\Lambda, \theta)+f(\Lambda, \theta)-\frac{\left(1-a^{2}\right)}{\tau_{R}\left(1+a^{2}-2 a \cos 2 \theta\right)^{1 / 2}}=0$,

$F_{0}(-\Lambda, \theta)+f(-\Lambda, \theta)-1=0$,

$f(z, \theta)=f(z, \theta+2 \pi)$,

where if $\left(F_{0}(z, \theta), P_{0}\right)$ were an exact solution of the problem, in Eqs. (10)-(14) some terms would cancel each other, but all terms have been retained because $\left[F_{0}(z, \theta), P_{0}\right]$ will only be an approximation to the solution in the iterative scheme.

A finite difference scheme has been developed characterizing the mesh as the intersection between the free surface and the planes,

$$
\begin{aligned}
& z=\Lambda\left(\frac{2 j}{J}-1\right), \quad j=0,1, \ldots, J, \\
& \theta=\frac{2 \pi}{I+1} i, \quad i=0,1, \ldots, I .
\end{aligned}
$$

Doing so, the system (10)-(14) yield a linearized finitedifference equations system that can be written as follows:

$$
\begin{aligned}
& \alpha_{i j} f_{j}^{i}+\beta_{i j} f_{j-1}^{i}+\gamma_{i j} f_{j+1}^{i}+\delta_{i j} f_{j}^{i-1}+\varphi_{i j} f_{j}^{i+1} \\
&+\phi_{i j}\left(f_{j+1}^{i+1}-f_{j+1}^{i-1}-f_{j-1}^{i+1}+f_{j-1}^{i-1}\right)=-B z_{j}+p=\psi_{i j}, \\
& i=0, \ldots, I, \quad j=0, \ldots, J, \\
& f_{J}^{i}= \frac{\left(1-a^{2}\right)}{\tau_{R}\left(1+a^{2}-2 a \cos 2 \theta_{i}\right)^{1 / 2}}-F_{0, J}^{i}, \quad i=1, \ldots, I, \\
& f_{0}^{i}=1-F_{0,0}^{i}, \quad i=1, \ldots, I, \\
& \sum_{i=0}^{I} \sum_{j=0}^{J} a_{i j} f_{j}^{i}=A, \\
& f_{j}^{0}-f_{j}^{I+1}=0,
\end{aligned}
$$

where the coefficients $\alpha_{i j}, \beta_{i j}, \gamma_{i j}, \delta_{i j}, \varphi_{i j}, \phi_{i j}, \psi_{i j}, a_{i j}$, and $A$ are functions of the values $F_{0, j}^{i}$, and $P_{0}$. The resulting set of linear algebraic equations has been solved by Crout's method with partial pivoting.

If no further modifications were made, the algorithm would destabilize when crossing any critical point (both turning points and pitchfork points). To stabilize the algorithm, a new equation needs to be included. ${ }^{15-17}$ With the method modified in this way, a sequence of equilibrium shapes is obtained whether they are stable or unstable. The details of the numerical methods used to locate bifurcation and limit points in the families of equilibrium shapes are identical to those outlined elsewhere ${ }^{15,16}$ and will not be repeated here.

The extra equation added to stabilize the method defines the arclength parameter, but the arc's origin needs to be periodically actualized because of the error introduced with the discretization. To define the arc's origin a second known configuration is needed. The integration must therefore always start from two known configurations in order to define the starting point and the advancing direction along the equilibrium shapes sequence for a given value of the parameter $a$, 
which is a monotonous function of the eccentricity, and changing the Bond number, $B$. It is important to start the integration with known stable shapes because the numerical method can determine a change in the stability character but not the stability character. If starting from a stable equilibrium shape, the Bond number can be increased to obtain a sequence of stable equilibrium shapes until a maximum in $B$ is reached. Alternatively the Bond number can be decreased to obtain another sequence of stable equilibrium shapes until a minimum in $B$ is reached. Beyond the maximum and minimum values of the Bond number, the equilibrium shapes of both sequences are unstable. In both cases extremes are found because the unstabilities are turning points. In all the analyzed cases the instabilities are due to a turning point and never has a pitchfork point been encountered.

For small enough values of $a$ an analytical asymptotic expression ${ }^{13}$ for $B=0$ and the given value of $a$ suffices for one of the initial shapes, while the second equilibrium shape is an analytical asymptotic expression for the same value of $a$ and slightly different value of $B$. The same two initial shapes may be used as initial equilibrium shapes to advance in the opposite direction and reach the other stability limit interchanging their role. If the value of $a$ is not so small, the analytical approximations provide too bad of an approximation for the two initial shapes needed and the sequence never starts. A way to overcome this problem is to use equilibrium shapes already calculated with the numerical method for a slightly smaller value of $a$ and two different values of $B$. These solutions are then used without any change but assuming a slightly larger value of $a$. Note that not necessarily either of the two initial values of $B$ for the two shapes is zero as, if $a$ is large enough, the stable range of $B$ may not include zero for slenderness close to $\pi$. The range of stable Bond numbers decreases for increasing values of $a$ until it vanishes just in the same way as for the analytical asymptotic solution developed below.

To illustrate the above described behavior, a sequence of equilibrium shapes has been calculated for a particular case and represented in Figs. 2 and 3. For $\Lambda=2.9$ and $a=0.2$, the area of section $z=\Lambda / 2$ is plotted in Fig. 2 versus the Bond number. For this disk configuration two turning points are encountered. This value of the parameter measuring the eccentricity, $a=0.2$, and the selected slenderness, $\Lambda=2.9$, yield stable configurations, provided the values of the Bond number lie in the stable region, which is the one bounded by the mentioned turning points. The value of $B$ for these two particular points is extracted and later used for plotting the stability limits for every value of $a$, as can be seen in Fig. 4 .

As the value of $a$ increases, the distance between the two turning points decreases until the stable branch collapses in an inflection point. The evolution of the area of section $z$ $=\Lambda / 2$ with the Bond number for a liquid bridge configuration having $\Lambda=2.9$ and $a=0.7$ is shown in Fig. 3. No turning points exist for this value of $a$ and a single unstable branch is obtained.

Figures 2 and 3 also show the interface shape of equilibrium liquid bridges for several Bond values corresponding to different points along the curves. Note that, from all plotted shapes, only (b), (c), and (d) of Fig. 2 are stable equilib-

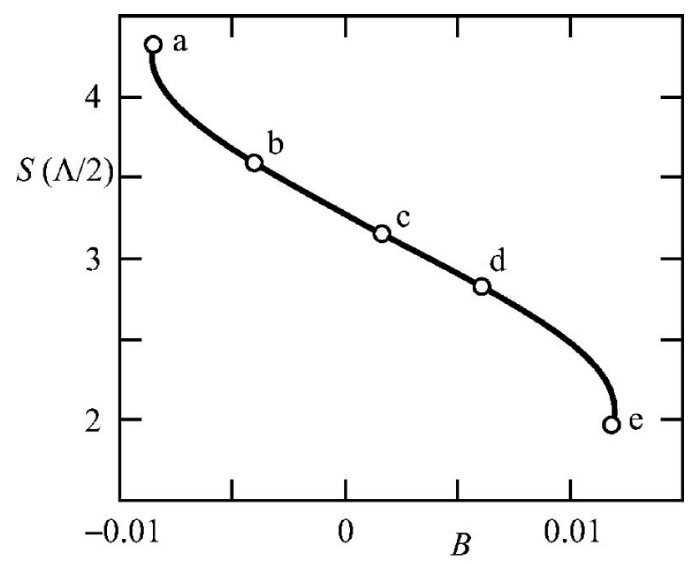

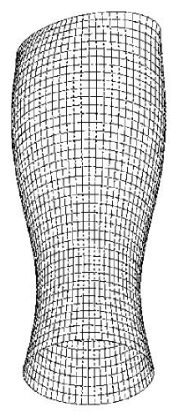

a

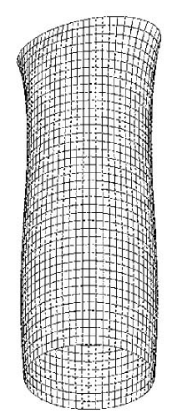

d

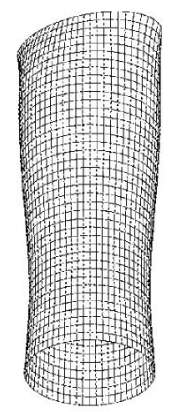

b

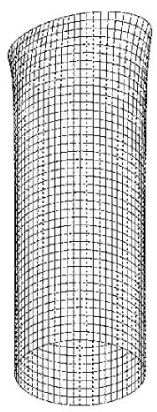

c
FIG. 2. Evolution with the Bond number $B$ of the cross-section area at a quarter of the total length of a liquid bridge for a disk configuration $(\Lambda=2.9$, $a=0.2$ ) with a stable range of Bond numbers bounded by unstable regions. The equilibrium shapes for some fluid configurations (identified by letters on the curve) are drawn below. Shapes b, c, and d are stable, and a and e are unstable.

rium shapes, while the rest are unstable equilibrium shapes, as explained above.

Figure 4 shows the stability limits in the $a-B$ plane for different slendernesses. A detail has been plotted in Fig. 5 to show the behavior near the Rayleigh stability limit $(\Lambda=\pi)$. Qualitatively, all limits behave as parabolas for small values of $a$, but the slopes of the lower limit are greater than those of the upper limit and, finally, both curves contact in a point; beyond that point there are no stable configurations. The known asymptotic approximation does not fit well the numerical results when $B$ and especially $a$ are not very small, because the stable region found numerically is far smaller than the asymptotic one; thus a higher-order approximation is developed in the next section. 


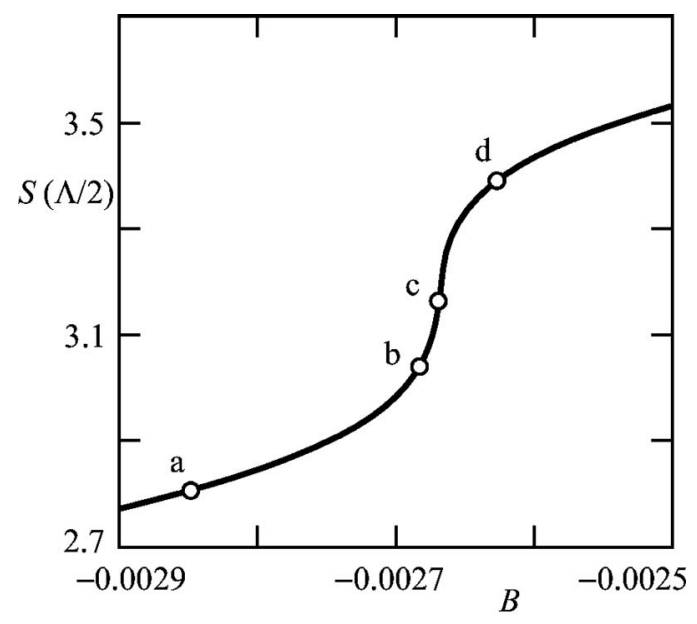

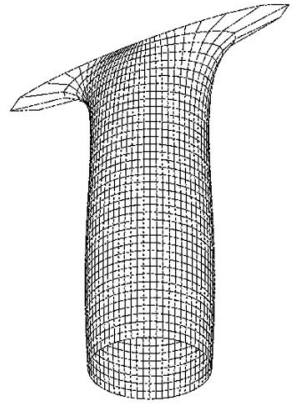

a

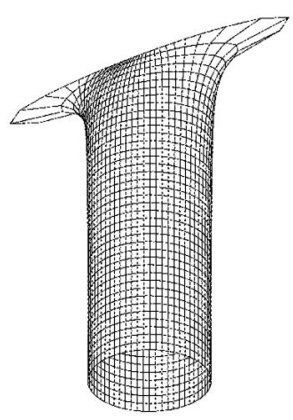

c

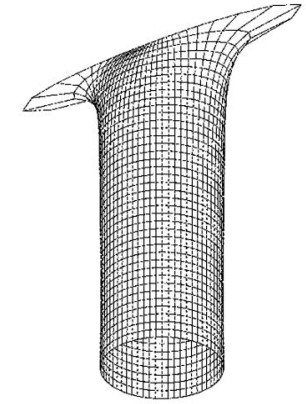

b

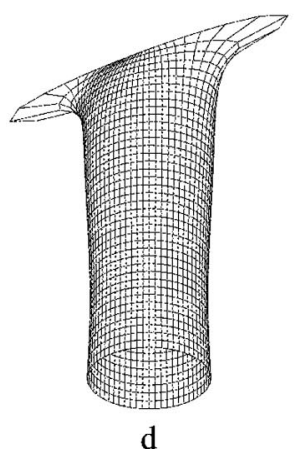

d
FIG. 3. Evolution with the Bond number $B$ of the cross-section area at a quarter of the total length of a liquid bridge for a disk configuration $(\Lambda=2.9$, $a=0.7$ ) with no stable range of Bond numbers. The equilibrium shapes for several fluid configurations are drawn below. All the equilibrium shapes are unstable and they are identified by letters on the curve.

\section{ASYMPTOTIC ANALYSIS}

The boundary conditions at the disks can be expressed in dimensionless form as

$$
\begin{aligned}
& F(\Lambda, \theta)=F^{+}(\theta)=1+\sum_{n=1}^{\infty}\left(\gamma_{n}^{+} \cos n \theta+\delta_{n}^{+} \sin n \theta\right), \\
& F(-\Lambda, \theta)=F^{-}(\theta)=1,
\end{aligned}
$$

where the lower disk is already specialized for a circular shape. If the upper disk has a symmetry axis in $\theta=0$, all coefficients $\delta_{n}^{+}$are zero.

As it is well known, in the case of liquid bridges in gravitationless conditions $(B=0)$, spanning between coaxial, equal in diameter, circular disks $\left(H=\gamma_{n}^{+}=\delta_{n}^{+}=0\right)$, and having cylindrical volume $(V=1)$, the problem under

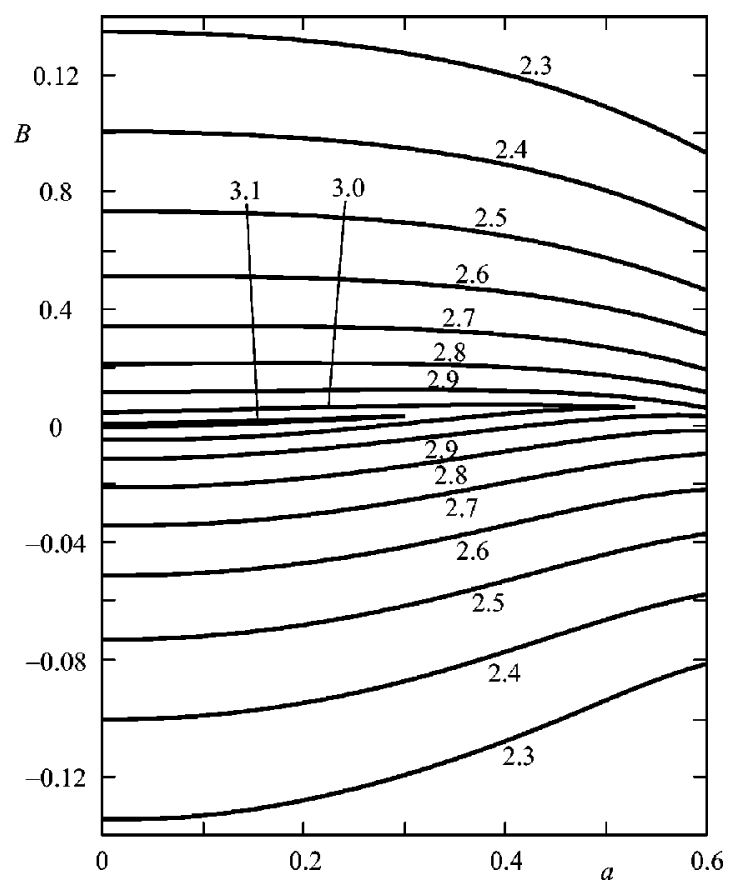

FIG. 4. Stable configurations, Bond number range as a function of $a$. Labels identify the value of the slenderness $\Lambda$. A detail for slendernesses close to $\pi$ and smaller values of $B$ is shown in Fig. 5 .

consideration has the trivial equilibrium solution $F(z, \theta)$ $=1, P=1$ for any $\Lambda$. The introduction of the expansions $F(z, \theta)=1+\epsilon \hat{f}(z, \theta)+O\left(\epsilon^{2}\right) \quad$ and $\quad P=1+\epsilon \hat{p}+O\left(\epsilon^{2}\right)$, where $\epsilon$ stands for the magnitude of the deformation of the interface, allows us to calculate $\hat{f}(z, \theta)$ after neglecting $O\left(\epsilon^{2}\right)$ terms. Nontrivial (different of zero) solutions of the linear problem appear only for a discrete number of values of $\Lambda$. The smallest value of $\Lambda$ for which a nontrivial solution

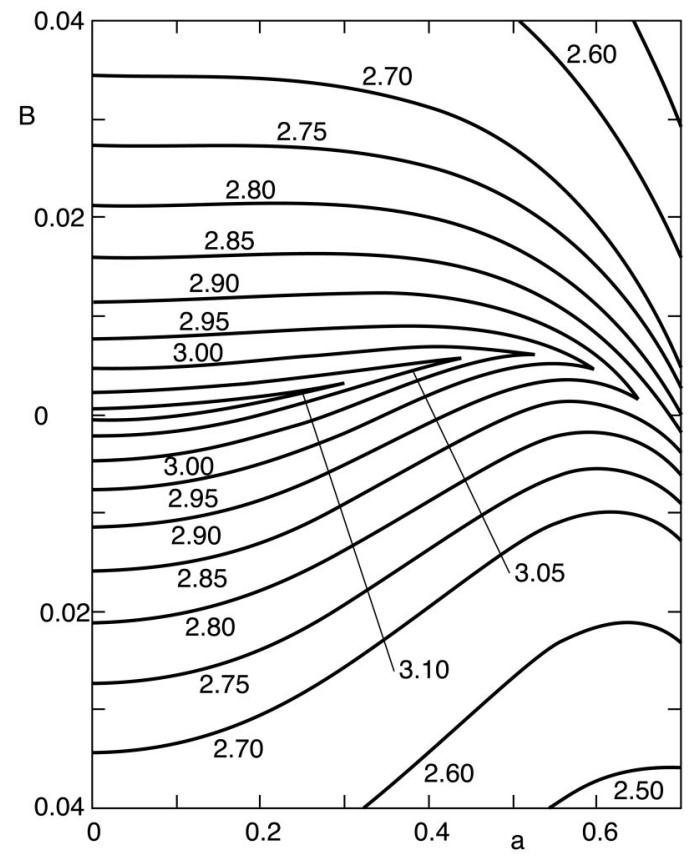

FIG. 5. Stable configurations, Bond number range as a function of $a$ for slendernesses close to $\pi$. Labels identify the value of the slenderness $\Lambda$. 


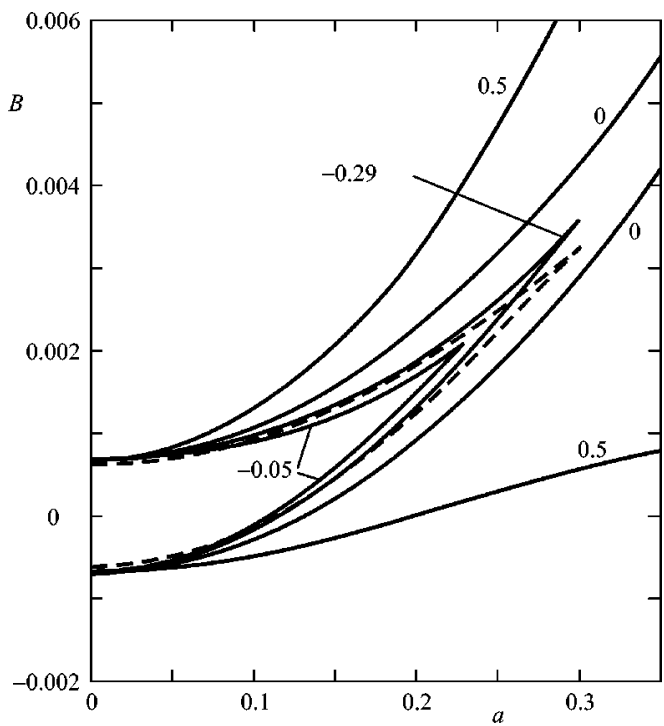

FIG. 6. Bond number at the stability limit, $B$, versus the parameter used to measure the ellipse eccentricity, $a$, according to expression (27). The figure has been calculated for $\Lambda=3.1$. Numbers on the curves indicate the value of $k$. A curve labeled with $k=0$ reproduces the results of expression (24). The dashed line corresponds to the numerical results.

(bifurcation to noncylindrical equilibrium shapes) exists is for $\Lambda=\pi$ (the well-known Rayleigh stability limit), where the transition from stable to unstable equilibrium shapes occurs. Therefore, the only relevant instability appears at $\Lambda=\pi$ and, within this approximation, the departure from the cylinder of the unstable equilibrium shapes are defined by $\hat{f}(z, \theta)=\sin (\pi z / \Lambda), \hat{p}=0$, which is the only solution of the linear problem that has to be considered in order to calculate the variation of the maximum stable slenderness.

For the problem stated here, the bifurcation equation reduces to ${ }^{13}$

$$
2 B+2 \epsilon \lambda-\frac{\Gamma^{+}}{4 \pi}-\frac{3}{2} \epsilon^{3}+\cdots=0,
$$

where

$$
\Gamma^{+}=\sum_{n=1}^{\infty}\left[\left(\gamma_{n}^{+}\right)^{2}+\left(\delta_{n}^{+}\right)^{2}\right]=\frac{1}{\pi} \int_{0}^{2 \pi}[F(\pi, \theta)-1]^{2} \mathrm{~d} \theta .
$$

If the elliptical disk is only slightly eccentric, $a \ll 1$, the boundary condition at this disk can be expressed as

$$
\begin{aligned}
F(\pi, \theta) & =\left(1-a^{2}\right)\left(1+a^{2}-2 a \cos 2 \theta\right)^{-1 / 2} \\
& \approx 1+a \cos 2 \theta+O\left(a^{2}\right),
\end{aligned}
$$

and then $\Gamma^{+}=a^{2}+O\left(a^{4}\right)$. Hence, for a given value of the maximum slenderness $\Lambda_{\text {max }}$, the variation with the parameter measuring the ellipse eccentricity, $a$, of the Bond number for which the liquid bridge becomes unstable, is given by

$$
B= \pm \frac{4}{9}\left(1-\frac{\Lambda_{\max }}{\pi}\right)^{3 / 2}+\frac{1}{8 \pi} a^{2}
$$

Equation (24) has been represented in Fig. 6 (the lines labeled with zero). In this plot two branches are shown, depending on the sense of the gravity vector for $\Lambda=3.1$. Let us consider first the case of two circular disks $(a=0)$ and $B$ $>0$ (upper branch). The deformation of the liquid bridge interface due to gravity will be a bulging of the liquid column in its lower half and a necking at the upper one. There is a value of the Bond number for which the interface shape becomes unstable and the breaking of the liquid column occurs if the value of the Bond number increases.

When an elliptical disk is considered (the upper one), there is an excess of volume close to the noncircular disk, so that the liquid column necks closer to the circular disk. Therefore, in this case (gravity pointing to the circular disk) the interface deformation due to the existence of an elliptical disk is in opposition to the interface deformation due to gravity, so that the value of the Bond number needed to break the liquid bridge will be larger as the value of the parameter $a$ grows. Points above the stability limit curve in the plane $a$ $-B$ represent unstable configurations, whereas points under this curve, if no other perturbations are taken into account, represent stable liquid bridge configurations.

When gravity points to the noncircular disk (the leftmost part of the lower curve), the interface deformation caused by both effects is similar (in both cases the liquid column necks at the bottom half and bulges at the upper one). Since both effects act in the same sense, if one of them grows the second must be reduced to keep the liquid bridge configuration stable. Therefore, in this branch points above the curve represent stable configurations while the unstable ones are represented by points lying below the curve. The same tendency of the curve holds even when, for larger values of $a$, the gravity points to the circular disk. If both branches are considered, obviously the stable region is that located between both curves, whereas points outside this region represent unstable liquid bridges.

Expression (24) predicts that the influence of $a$ on the maximum stable Bond is quadratic and equal for both branches. This result is only representative for small values of $a$ and $B$. For large values of the parameters, this simple asymptotic result cannot reproduce the behavior of the configuration. Within this approximation both branches never intersect, therefore $a$ can take values as large as desired, provided that $B$ has the appropriate value, and two possible extremes of $B$ will exist no matter the value of $a$. Note that $a$ is bounded by 1 , for that particular value the disk collapses to a line. Obviously, the behavior described by Eq. (24), asymptotically correct for small values of all parameters, cannot hold for large values of $a$ (the critical point depending on the slenderness), where the two mentioned extremes should disappear and a new situation with no stable interval of $B$ should occur. Higher-order terms have to be retained to reproduce the phenomena. From Eq. (21), it is deduced that $B$ and $a^{2}$ are of the order of $\epsilon^{3}$, and $\lambda$ of the order of $\epsilon^{2}$. All terms considered in the equation are of order $\epsilon^{3}$ and the first terms neglected are of order $\epsilon^{4}$. To reproduce the numerical behavior described above only new terms in $\epsilon^{4}$ need to be considered and there is no need to consider higher-order tems. A symmetry analysis shows that the only nonzero $\epsilon^{4}$ term is that in $a^{2} \epsilon$, hence the new bifurcation equation reads as 


$$
2 B+2 \epsilon \lambda-\frac{1}{4 \pi} a^{2}-\frac{3}{2} \epsilon^{3}+k a^{2} \epsilon+\cdots=0 .
$$

The analytical calculation of the value of the new coefficient, $k$, multiplying the new term in $a^{2} \epsilon$ is too involved, so its value will be estimated by fitting the analytical results to the numerical results. Figure 6 shows how the stability limits look like for different values of $k$ along with the exact numerical result.

To obtain the stability limit, one should look for possible extremes of $B$ as a function of $\epsilon$ for given values of the other parameters $(a$ and $\lambda$ ). After the derivation of Eq. (25), one finds

$$
2 \lambda-\frac{9}{2} \epsilon_{\mathrm{ext}}^{2}+k a^{2}+\cdots=0 .
$$

The values of $\epsilon_{\text {ext }}$ (the deformation at the stability limit) depend not only on $\lambda$ but also on $a$. Thus, assuming a stable shape for $a=0$ and a negative value of $k$, and increasing the value of $a$, one reaches a critical value of $a, a_{\text {crit }}$; beyond that value no further solutions for $\epsilon_{\text {ext }}$ exist. After substitution of $\epsilon_{\text {ext }}$ in Eq. (25), one finds two extreme values of $B$ for $a<a_{\text {crit }}$, one for $a=a_{\text {crit }}$ and none for $a>a_{\text {crit }}$,

$$
B=\frac{1}{8 \pi} a^{2} \mp \frac{4}{9}\left(\lambda+\frac{1}{2} k a^{2}\right)^{3 / 2} \text {. }
$$

It is also easy to show that the curves yielding $B$ as a function of $a$ are tangent at $a=a_{\text {crit }}$. This behavior is exactly the one obtained with the numerical analysis described in the preceding section.

The qualitative behavior of the numerical results is now explained by the asymptotic analysis, even though the parameter $k$ has not been calculated. Taking into account the results given by Eq. (27), a value for $k$ can be obtained, fitting the asymptotic results to the numerical ones. The intersection point, where the stability region ends, is adjusted to coincide with the numerical results for $\Lambda=3.1$; doing so, the obtained value of $k$ is -0.29 . The analytical results for $\Lambda=3.1$ and $k=-0.29$ are plotted along with the numerical results in Fig. 6.

\section{CONCLUSIONS}

To summarize, the combined effect of Bond number and disk shape has been analyzed numerically. Particular shapes of the disks (an ellipse and a circumference) have been considered and the stability limits have been obtained for a wide range of slendernesses. These slendernesses result in large values of $a$ for small values of $B$ and, for those values, the $\epsilon^{3}$-order asymptotic analysis no longer yields accurate enough results. Nevertheless, it is demonstrated that just by retaining one more term in the bifurcation equation, the behavior of the solutions obtained numerically are qualitatively reproduced by the asymptotic analysis.

\section{ACKNOWLEDGMENTS}

The authors are indebted to Professor J. M. Perales and the referees for their useful comments.

${ }^{1}$ A. Eyer, H. Leiste, and R. Nitsche, "Floating zone growth of silicon under microgravity in a sounding rocket," J. Cryst. Growth 71, 173 (1985).

${ }^{2}$ I. Martínez and A. Cröll, "Liquid bridges and floating zones," in Proceedings of the VIII European Symposium on Materials and Fluid Sciences in Microgravity, ESA SP-333, 1992, p. 135.

${ }^{3}$ L. A. Slobozhanin and J. M. Perales, "Stability of liquid bridges between equal disks in an axial gravity field," Phys. Fluids A 5, 1305 (1993).

${ }^{4}$ L. A. Slobozhanin and J. I. D. Alexander, "Combined effect of disk inequality and axial gravity on axisymmetric liquid bridge stability," Phys. Fluids 10, 2473 (1998).

${ }^{5}$ B. J. Lowry and P. H. Steen, "Stability of slender liquid bridges subjected to axial flows," J. Fluid Mech. 330, 189 (1997).

${ }^{6}$ M. J. Marr-Lyon, D. B. Thiessen, and P. L. Marston, "Stabilization of a cylindrical capillary bridge far beyond the Rayleigh-Plateau limit using acoustic radiation pressure and active feedback," J. Fluid Mech. 351, 345 (1997).

${ }^{7} \mathrm{H}$. González and A. Castellanos, "The effect of residual gravity on the stability of liquid columns subjected to electric fields," J. Fluid Mech. 249, 185 (1993).

${ }^{8}$ M. P. Mahajan, S. Zhang, M. Tsinge, P. L. Taylor, and C. Rosenblatt, "Stability of magnetically levitated liquid bridges of arbitrary volume subjected to axial and lateral gravity," J. Colloid Interface Sci. 213, 592 (1999).

${ }^{9}$ I. E. Parra, J. M. Perales, and J. Meseguer, "On the influence of Marangoni convection on the stability of liquid bridge interfaces," Adv. Space Res. 29, 625 (2002).

${ }^{10} \mathrm{~V}$. Luengo, J. Meseguer, and I. E. Parra, "Experimental study of the stability of long, axisymmetric liquid bridges between solid supports at different temperatures," Exp. Fluids 34, 412 (2003).

${ }^{11}$ I. Martínez, J. Meseguer, and J. M. Perales, "Analytical modeling of floating zone crystal growth,” Adv. Space Res. 29, 569 (2002).

${ }^{12}$ I. Martínez, J. Meseguer, and J. M. Perales, "Simulation of nonaxisymmetric floating zone crystal growth under microgravity," Microgravity Sci. Technol. 13/2, 3 (2002).

${ }^{13}$ J. Meseguer, J. M. Perales, and J. I. D. Alexander, "A perturbation analysis of the stability of long liquid bridges between almost circular supporting disks," Phys. Fluids 13, 2724 (2001).

${ }^{14}$ J. F. Geer and J. C. Strikwerda, "Vertical slender jets with surface tension," J. Fluid Mech. 135, 155 (1983).

${ }^{15}$ A. Laverón-Simavilla and J. M. Perales, "Equilibrium shapes of nonaxisymmetric liquid bridges of arbitrary volume in gravitational fields and their potential energy," Phys. Fluids 7, 1204 (1995).

${ }^{16}$ A. Laverón-Simavilla and E. Checa, "Effect of a lateral gravitational field on the nonaxisymmetric equilibrium shapes of liquid bridges held between eccentric disks and of volumes equal to those of cylinders," Phys. Fluids 9, 817 (1997).

${ }^{17}$ H. B. Keller, Lectures on Numerical Methods in Bifurcation Problems (Springer-Verlag, Berlin, 1987). 